\title{
Increased expression of PHD3 represses the HIF-1 signaling pathway and contributes to poor neovascularization in pancreatic ductal adenocarcinoma
}

\author{
Takayuki Tanaka $\cdot$ Tao-Sheng Li $\cdot$ Yoshishige Urata $\cdot$ Shinji Goto $\cdot$ \\ Yusuke Ono • Miho Kawakatsu • Hajime Matsushima • Masataka Hirabaru • \\ Tomohiko Adachi · Amane Kitasato • Mitsuhisa Takatsuki - Tamotsu Kuroki • \\ Susumu Eguchi
}

Received: 16 October 2014/Accepted: 5 December 2014/Published online: 27 December 2014

(C) The Author(s) 2014. This article is published with open access at Springerlink.com

\begin{abstract}
Background Pancreatic ductal adenocarcinoma (PDAC) is known as one of the most malignant potential diseases with poor neovascularization. By comparing PDAC to hepatocellular carcinoma (HCC), which is well vascularized, we investigated the mechanisms and tumor biological significance of the poor neovascularization in PDAC.

Methods Surgical specimens from primary PDAC and HCC patients were immunohistologically stained to detect the expressions of CD105, CD44, HIF-1 $\alpha$, PHD3, and Siah2. We also used two PDAC and two HCC cell lines to compare the expressions of HIF-1 $\alpha$, PHD3, and CD44, as well as the production of VEGF in hypoxic condition. The role of PHD3 in regulating HIF-1 $\alpha$ expression was further confirmed by siRNA knockdown in a PDAC cell line that highly expressed PHD3.
\end{abstract}

T. Tanaka · T.-S. Li · Y. Urata - S. Goto · Y. Ono ·

M. Kawakatsu

Department of Stem Cell Biology, Atomic Bomb Disease

Institute, Nagasaki University, 1-12-4 Sakamoto,

Nagasaki 852-8523, Japan

e-mail: litaoshe@nagasaki-u.ac.jp

Y. Urata

e-mail: urata@nagasaki-u.ac.jp

S. Goto

e-mail: sgoto@nagasaki-u.ac.jp

Y. Ono

e-mail: yusuke-ono@nagasaki-u.ac.jp

M. Kawakatsu

e-mail: mkawa@nagasaki-u.ac.jp

T. Tanaka $(\bowtie) \cdot$ H. Matsushima $\cdot$ M. Hirabaru $\cdot$ T. Adachi ·

A. Kitasato · M. Takatsuki - T. Kuroki - S. Eguchi

Department of Surgery, Nagasaki University Graduate School of

Biomedical Sciences, 1-7-1 Sakamoto, Nagasaki 852-8501,

Japan

e-mail: pay-it-forward.197675@hotmail.co.jp
Results There were significantly fewer microvessels but more cancer stem cells in PDAC specimens compared to HCC specimens. The expression of CD105 was reversely related to the expression of CD44 in PDAC and HCC specimens. PDAC specimens also showed higher expressions of PHD3 but lower expressions of HIF-1 $\alpha$. Similarly, the expression of PHD3 was observed clearly in PDAC cell lines, but was almost completely negative in HCC cell lines. Hypoxic stimulation clearly enhanced HIF- $1 \alpha$ expression and VEGF secretion in both HCC cell lines, but did not significantly change in PDAC cell lines. The knockdown of PHD3 in PDAC cells restored the hypoxicinduced HIF- $1 \alpha$ expression, which accordingly stimulated the cells' VEGF secretion.

\author{
H. Matsushima \\ e-mail: h.matsushima.1020@ever.ocn.ne.jp \\ M. Hirabaru \\ e-mail: m.hirabaru@hotmail.co.jp \\ T. Adachi \\ e-mail: adatomo@nifty.com \\ A. Kitasato \\ e-mail: a-kit@bg8.so-net.ne.jp \\ M. Takatsuki \\ e-mail: takapon@nagasaki-u.ac.jp \\ T. Kuroki \\ e-mail: tkuroki-gi@umin.ac.jp \\ S. Eguchi \\ e-mail: sueguchi@nagasaki-u.ac.jp
}


Conclusions The enhanced expression of PHD3 might likely contribute to the poor neovascularization and affect the biological characterization in PDAC cancer cells.

Keywords HIF-1 $\alpha$ - PDH3 - CD44 - VEGF - Pancreatic ductal adenocarcinoma

\section{Introduction}

Pancreatic ductal adenocarcinoma (PDAC) is well known as one of the most lethal malignant diseases, showing poor responses to various therapies [1-6]. Compared to other solid tumors such as hepatocellular carcinomas (HCCs), which are well vascularized, the majority of PDAC cases are identified as a hypovascular mass by medical imaging examinations. As tumor hypoxia has been demonstrated to correlate with resistance to therapeutics and increased risks of invasion and metastasis [7-10], it is critical to understand the molecular mechanisms and tumor biological significance of the poor neovascularization in PDACs.

Hypoxia-inducible factor-1 (HIF-1) is the major regulator of oxygen homoeostasis, which consists of two subunits: the hypoxic sensitive HIF-1 $\alpha$ subunit and the constitutively expressed HIF-1 $\beta$ subunit [11-14]. Generally, tumor hypoxia activates HIF- $1 \alpha$ to induce the transcription of vascular endothelial growth factor (VEGF) and neovascularization for supporting cell survival and invasion [1-13]. Three prolyl hydroxylase domain-containing proteins (PHD, PHD1, PHD2, and PHD3) are functionally known as cellular oxygen sensors in the mediation of the oxygen-dependent degradation of HIF-1 $\alpha$ [15-17]. PHD3 is widely expressed in many types of tissues, including those of cancer cells. In response to hypoxia, PHD3 is activated to serve as a negative feedback mechanism of neovascularization [18]. It was demonstrated that PHD3 is upregulated in human pancreatic cancer and plays critical roles in tumor growth and neovascularization [19, 20], and it was also reported that the E3 ubiquitin-protein ligase Siah2 mediates the degradation of HIF-1 $\alpha$ [21]. However, the molecular mechanism underlying the poor neovascularization in PDACs has been unclear.

Cancer stem cells (CSCs) have been identified in various types of solid tumors, including PDACs and HCCs. These CSCs are known to play a key role in recurrence and metastasis because of their biological property of high resistance to hypoxia and various therapies. CD44, one of the cell-surface markers commonly used for identifying CSCs, was shown to interact with PKM2 to enhance the glycolytic of cancer cells [22-24], which results in increased glutathione levels, thereby contributing to resistance to hypoxia and drug. In light of the biological properties of CSCs, a relationship among poor neovascularization, CSCs and therapeutic resistance is not unlikely. By using well-vascularized HCCs as a control, we herein tried to identify the tumor biological properties and uncover the molecular mechanism underlying the poor neovascularization of PDACs.

\section{Methods}

Surgical specimens of PDAC and HCC

To narrow the variation in the analyses, we selected 12 surgically resected tissue samples of PDAC with the tumor diameter of 2-4 cm only from patients who had undergone surgery between April 2011 and April 2012, and we selected 11 surgically resected tissue samples of HCC with the tumor diameter of $2-4 \mathrm{~cm}$ from patients who had undergone surgery for HCC between April 2010 and December 2011 at Nagasaki University Hospital.

All 23 of the resected specimens were fixed in $10 \%$ formalin. The specimens were then sectioned, and serial sections were cut from paraffin blocks for the immunohistochemical analysis. This study was approved by the Ethics Review Board for Clinical Research at Nagasaki University, and conducted in accordance with the Declaration of Helsinki.

\section{Immunohistochemical analysis}

Tissue sections were deparaffinized by ethanol concentrations and washed in phosphate-buffered saline (PBS). The sections were then treated with hot water $\left(95^{\circ} \mathrm{C}\right)$ for citric acid buffer for $20 \mathrm{~min}$. After being washed with PBS, the samples were treated with $0.03 \%$ hydrogen peroxide in methyl alcohol and thereafter with $0.25 \%$ casein in PBS containing stabilizing protein and $0.015 \mathrm{~mol} / \mathrm{L}$ sodium azide (Protein Block, Serum-Free, Dako, Japan) at room temperature for $20 \mathrm{~min}$.

The sections were incubated with mouse monoclonal antibodies against human HIF1 $\alpha$ (Novus Biologicals, Littleton, CO, USA), PHD3 (Abcam Discover More, Tokyo, Japan), CD44 (Leica Biosystems Newcastle, UK), CD105 (R\&D Systems, Minneapolis, MN), and human Siah2 (Novus Biologicals), all with 1:100 dilution in PBS at room temperature for $120 \mathrm{~min}$. After rinsing with PBS, the sections were treated with a rabbit polyclonal antibody against mouse $\operatorname{IgG}, \operatorname{IgA}$, and $\operatorname{IgM}$ at room temperature for $60 \mathrm{~min}$ and washed with PBS. The peroxidase/antiperoxidase complex was allowed to react with the mouse and rabbit antibody, and the sections were then stained with 3,3-diaminobenzidine tetrahydrochloride (DAB) containing $0.03 \%$ hydrogen peroxide [Envison kit/HRP (DAB), Dako, Japan]. Counterstaining was done with Mayer's hematoxylin. 
We quantitatively calculated the expressions of CD105 and CD44 by using imaging analysis software (WinROOF ver. 5.50, MitaniCorp., Fukui, Japan), and the rate of the positively stained areas was used for statistical analysis. The expressions of HIF1 $\alpha$, PHD3, and Siah2 were evaluated by counting the positively stained cells in high-power fields $(40 \times)$, and then graded on an arbitrary scale of (negative; no positively stained cells at all), \pm (pluses and minuses; 1-20 positively stained cells per field), or + (positive; over 20 positively stained cells per field).

Cell lines and cell culture

Two PDAC lines (HS766T and Panc-1) and two HCC cell lines (HepG2 and HuH7) purchased from the Japanese Collection of Research Bioresources (Osaka, Japan) were used for the experiments. Dulbecco's modified Eagle's medium (Sigma Aldrich, St. Louis, MO, USA) supplemented with $10 \%$ fetal bovine serum (FBS) was used for the cell cultures, and the medium was replaced with fresh medium every $48-72 \mathrm{~h}$. All cultures were maintained in a $5 \% \mathrm{CO}_{2}$ air-humidified atmosphere at $37{ }^{\circ} \mathrm{C}$.

\section{Western-blot analysis}

To compare the expressions of HIF-1 $\alpha$ and PHD3 in the PDAC and HCC cell lines, we grew cells in six-well plates to $\sim 70 \%$ confluence and then randomly separated them for a further 24-h incubation in a typical $\mathrm{CO}_{2}$ incubator $\left(\sim 20 \% \mathrm{O}_{2}\right)$ or in a $1 \% \mathrm{O}_{2}$ incubator in which the oxygen concentrations were balanced by nitrogen gas (Sanyo, Tokyo), respectively. After the medium was removed, the cells were washed twice with pre-chilled PBS $(\mathrm{pH} 7.4$; Invitrogen, Paisley, UK), and the monolayer was scraped into $150 \mu \mathrm{l}$ of lysis buffer (Mammalian Cell Lysis-1 Kit; Sigma Aldrich). The total cell lysates were centrifuged at $10,000 \times g$ for $10 \mathrm{~min}$, and the supernatants were analyzed by immunoblots. Equal amounts of protein $(30 \mu \mathrm{g})$ were separated by $10 \%$ sodium dodecyl sulfate-polyacrylamide gel electrophoresis (SDS-PAGE). Immunoblots were probed with mouse monoclonal antibodies against human HIF$1 \alpha$ and PHD3 followed by secondary antibody, and immunoreactive bands were visualized using 5-bromo-4chloro-3-indolyl phosphate (BCIP)/nitro blue tetrazolium (NBT).

\section{ELISA}

To measure the VEGF secretion from the PDAC and HCC cell lines, we grew the cells in six-well plates to $\sim 70 \%$ confluence. After changing the culture medium, we randomly selected cells for a further 24-h incubation in normoxia $\left(\sim 20 \% \mathrm{O}_{2}\right)$ or hypoxia $\left(\begin{array}{lll}\sim & \% & \mathrm{O}_{2}\end{array}\right)$ conditions. We collected the supernatants and measured the levels of VEGF by using an ELISA kit (R\&D Systems).

Flow cytometry

All cells were seeded in a six-well plate. After a 24-h incubation in normoxia and hypoxia conditions, the cells were trypsinized and labeled with an FITC-conjugated anti-mouse CD44 antibody (BD Biosciences, Franklin Lakes, NJ, USA) for $45 \mathrm{~min}$. Respective isotype controls were used as a negative control. After washing the cells, we performed a quantitative flow cytometry analysis using a FACSCalibur (Becton-Dickinson, Lincoln Park, NY, USA). We analyzed the acquired data using Cell Quest software (Becton-Dickinson).

Depletion of PHD3 by synthetic small interfering RNAs (siRNAs)

Three individual PHD3-specific small interfering siRNAs were chemically synthesized with the sequences $5^{\prime}$ UCCACAUCUGCUAUGAAUGAUUUCC-3' (\#1), 5' UCCAGGUCCAGCCUCAUGAUGUGUC- $3^{\prime}$ (\#2), and $5^{\prime}-$ UAGAGGACCAGCCUGUCGAUGAGGG-3' (\#3) (Invitrogen). Stealth RNAi Negative Control (Invitrogen) was used as a negative control. After 24-h plating, the cells were transfected with $20 \mathrm{nM}$ PHD3-siRNAs or control siRNA using Lipofectamine Transfection Reagent RNAiMAX (Invitrogen) in accordance with the manufacturer's instructions. After $12 \mathrm{~h}$ of transfection, we randomly selected cells to incubate for another $24 \mathrm{~h}$ of innormoxia and hypoxia conditions, respectively, and then harvested them for Western-blotting analysis. The supernatants were also collected, and the levels of VEGF were determined by an enzyme-linked immunosorbent assay (ELISA; R\&D Systems).

Statistical analysis

We used the Mann-Whitney $U$ test to compare the CD105 and CD44 expressions in the PDAC and HCC cell lines. The association of CD105 expression with CD44 expression was evaluated by Spearman's rank correlation coefficient test. The HIF-1 $\alpha$, PHD3, and Siah2 expressions in the PDAC and HCC cells were compared by the Mantel trend test. The levels of VEGF treated with siRNA-control and siPHD3 were compared with the Mann-Whitney $U$ test. The data are expressed as the mean \pm SD. $p$ values $<0.05$ were accepted as significant. 


\section{Results}

Expressions of CD105 and CD44 in surgically resected tissues of PDAC and $\mathrm{HCC}$

The expression of CD105, a marker that is commonly used for labeling the endothelial cells of microvessels in tumors, was observed at an extremely weak level in the PDAC specimens, but strongly in the HCC specimens (Fig. 1a). Quantitative analysis showed that the rate of positively stained areas in the PDAC specimens was significantly lower than that of the HCC specimens ( $0.2 \pm 0.3$ vs. $2.7 \% \pm 1.8 \%$, respectively, $p<0.0001$; Fig. 1a). The expression of CD44, one of the most commonly used cellsurface markers for CSCs, was high in the PDAC specimens and much lower in the HCC specimens (Fig. 1b).

Quantitative analysis showed that the rate of positively stained areas was significantly higher in the PDAC specimens compared to the HCC specimens ( $8.6 \pm 3.8$ vs. $2.8 \pm 0.8 \%, p<0.001$; Fig. 1b). In addition, there was a significant negative correlation between CD105 and CD44 in both the PDAC and HCC specimens $(p<0.01$ in PDAC and $p<0.001$ in HCC, Fig. 1c).

Expressions of HIF-1 $\alpha$, PHD3, and Siah2 in surgically resected tissues of PDAC and $\mathrm{HCC}$

We used an immunochemical analysis to determine the expression of HIF-1 $\alpha$ (the major regulator of oxygen homoeostasis) in the PDAC and HCC specimens; the results are shown in Fig. 2a. Of the 12 PDAC specimens, 11 were negative for HIF-1 $\alpha$ and only one tissue sample showed \pm expression. In contrast, of the 11 HCC specimens, five showed positive expression, two showed \pm expression, and four showed negative expression. The statistical analysis indicated that the expression of HIF- $1 \alpha$ was significantly poorer in the PDAC specimens compared to the HCC specimens ( $p<0.05$, Fig. 2a).

We also investigated the expressions of PHD3 and Siah2, two factors that have been demonstrated to regulate the degradation of HIF-1 $\alpha$. Surprisingly, of the 12 PDAC specimens, the expression of PHD3 was expressed
Fig. 1 Immunohistological staining for the expressions of CD105 and CD44 in surgical specimens from PDAC and HCC patients. a The expression of CD105, a marker for neovascularization, was extremely low in the PDAC specimens compared to the HCC specimens. The quantitative data and results of the statistical analysis between the groups are shown in the lower bar graph. There was a significant difference with the vascularity between PDAC and HCC. b A higher expression of CD44, a marker for cancer stem cells was observed in the PDAC specimens compared to the HCC specimens. The quantitative data and results of the statistical analysis between the groups are shown in the lower bar graph. c There was a significant negative correlation between the expressions of CD105 and CD44 in each specimens, for both PDAC $(p<0.01$, left $)$ and HCC $(p<0.001$, right $)$
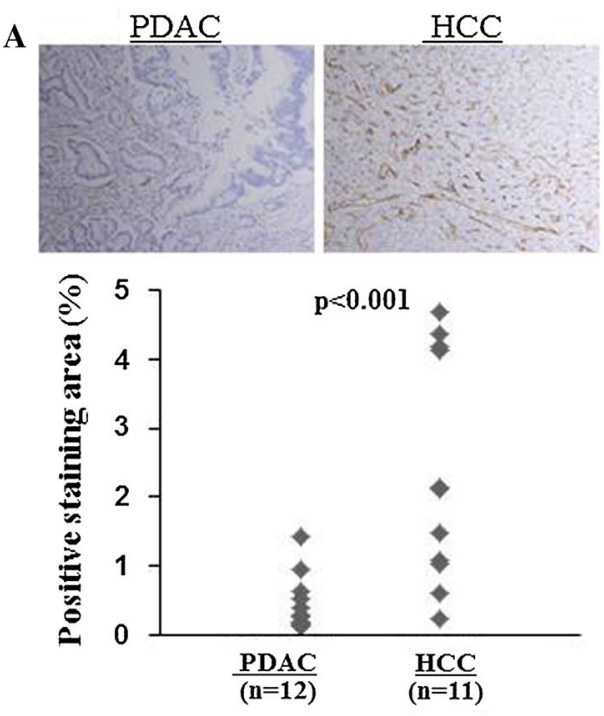

C

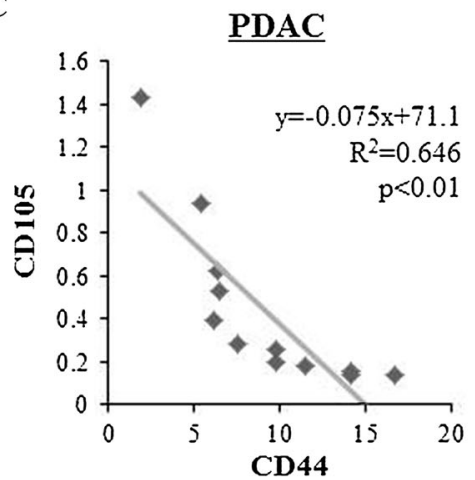

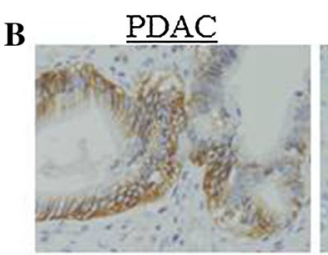
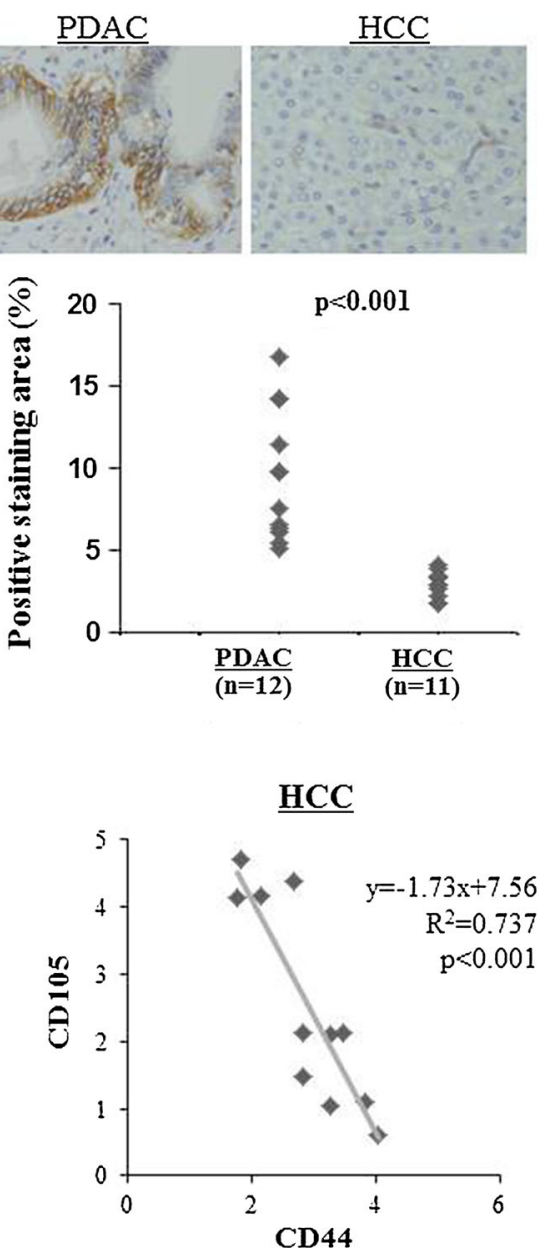
A

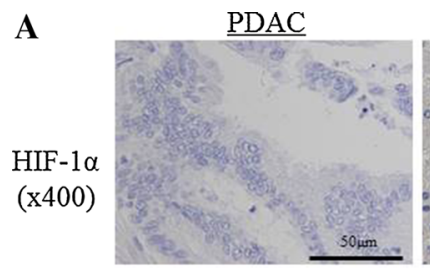

B
PHD3
$(\mathrm{x} 400)$
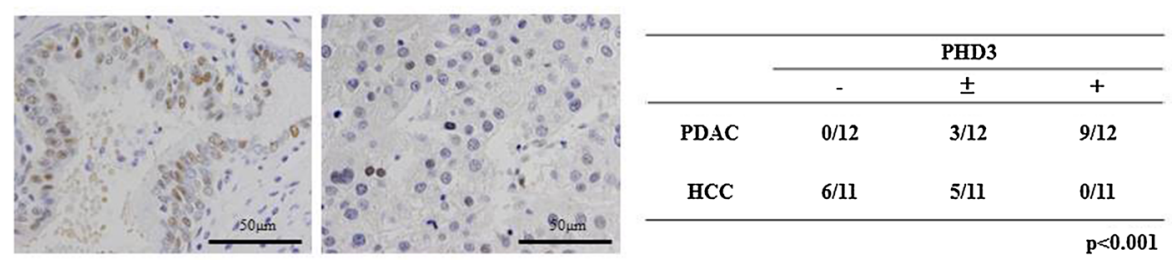

C
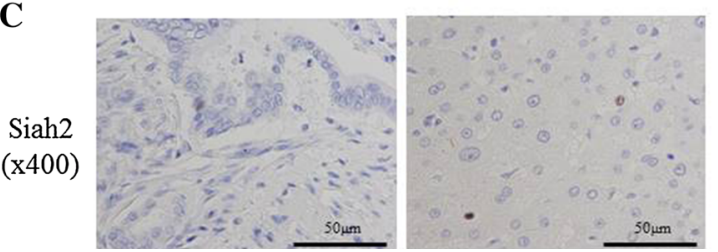

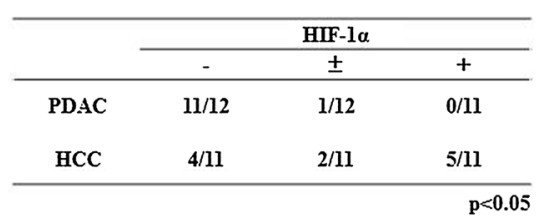

$\mathbf{p}<0.05$

\begin{tabular}{cccc}
\hline & \multicolumn{3}{c}{ Siah2 } \\
\cline { 2 - 4 } PDAC & $11 / 12$ & $1 / 12$ & $0 / 12$ \\
HCC & $6 / 11$ & $5 / 11$ & $0 / 11$ \\
\hline & & & $\mathrm{p}<0.05$
\end{tabular}

Fig. 2 Immunohistological staining for the expressions of HIF- $1 \alpha$, PHD3, and Siah2 in surgical specimens from PDAC and HCC patients. a HIF-1 $\alpha$ was positively stained in the nuclei of cancer cells in HCC specimens, but was negatively or extremely rarely stained in the PDAC specimens, and a semiquantitative analysis showed a significant difference between the groups $(p<0.05)$. b PHD3 was

positively in nine, weakly in three, and negatively in none. In contrast, only five of the $11 \mathrm{HCC}$ specimens showed a weak expression of PHD3, and no positive expression was found ( $p<0.001$, Fig. 2b). The expression of Siah2 was weakly observed in one of the 12 PDAC specimens and five of the 11 HCC specimens $(p<0.05$, Fig. 2c), and positive expression of Siah2 was found in neither PDAC nor HCC specimens.

Differing expressions of PHD3, HIF-1 $\alpha$, and CD44 between PDAC and HCC cell lines

We further investigated the mechanism as well as the tumor biological significance of the poor neovascularization in PDAC using two PDAC cell lines (HS766T and Panc-1) and two HCC cell lines (HepG2 and HuH7). In good agreement with the findings in surgically resected tissue samples of primary PDAC and HCC, a high expression of CD44 was observed in both of the PDAC cell lines, but the HCC cells were almost completely negative for CD44 (Fig. 3). The incubation of the cells in $1 \% \mathrm{O}_{2}$ for $24 \mathrm{~h}$ did not enhance the expression of CD44 in any of the cell lines.

A strong and clear expression of PHD3 was observed in the two PDAC cell lines, but PHD3 was negative or only slightly so in both of the HCC cell lines (Fig. 4a, b). The expression of Siah2 was also examined by Western-blot positively stained in many cancer cells in the PDAC specimens, but was negatively or weakly stained in the HCC specimens $(p<0.001)$. c The expression of Siah2 was negative or weak in both the PDAC and HCC specimens, but the semiquantitative analysis showed a significantly higher expression in the HCC specimens $(p<0.05)$

analysis. The expression of Siah2 was detected clearly in one PDAC cell line and one HCC cell line, but was extremely weak in the other PDAC and HCC cell lines (Fig. 4a, b). Interestingly, hypoxic stimulation by 24-h incubation in $1 \% \mathrm{O}_{2}$ upregulated the expression of HIF-1 $1 \alpha$ in both HCC cell lines, but not in the PDAC cell lines, although the basic expression level in the normoxic condition was comparable among all four cell lines (Fig. 4a, b). Moreover, although the VEGF secretion was comparable among all four cell lines in $20 \% \mathrm{O}_{2}$, the VEGF secretion in $1 \% \mathrm{O}_{2}$ was largely enhanced from both of the HCC cell lines, but only slightly increased from the two PDAC cell lines (Fig. 5).

PHD3 plays a critical role in regulating the HIF- $1 \alpha$ signaling pathway and neovascularization

Considering that the expression of HIF- $1 \alpha$ and PHD3 was observed to be quite similar between the two PDAC cell lines of Panc-1 and HS766T (Fig. 4), we selected only HS766T cells to see how the knock-down of PHD3 could change the response of cells to hypoxic stimulation. We found that the siRNA knock-down of PHD3 expression in HS766T cells recovered the hypoxia-induced HIF- $1 \alpha$ expression as well as the hypoxia-enhanced VEGF secretion (Fig. 6), but it did not change the expression of HIF-1 $\alpha$ in the $20 \% \mathrm{O}_{2}$ condition. 
Fig. 3 The expression of CD44 in PDAC (HS766T and Panc-1) and $\mathrm{HCC}$ (HepG2 and $\mathrm{HuH} 7$ ) cell lines. Cells were cultured in $20 \% \mathrm{O}_{2}$ or $1 \% \mathrm{O}_{2}$ for $24 \mathrm{~h}$, and the expression of the CSC marker CD44 was then estimated by flow cytometry and compared to cancer stem cell markers by hypoxic stimulation. The expression of CD44 was much higher in the PDAC cell lines compared to the HCC cell lines, but the 24-h incubation in $1 \% \mathrm{O}_{2}$ did not change the expression of CD44 in any of the cell lines. The green line shows the expression of CD44 in hypoxia $\left(1 \% \mathrm{O}_{2}\right.$ for $24 \mathrm{~h}$ ) and the purple area shows it in normoxia $\left(20 \% \mathrm{O}_{2}\right.$ for $24 \mathrm{~h}$ )

Fig. 4 Western-blot analysis of the expressions of PHD3 and HIF-1 $\alpha$ in the PDAC (HS766T and Panc-1) and HCC (HepG2 and $\mathrm{HuH7}$ ) cell lines. a The expression of PHD3 was clearly detected in both of the PDAC cell lines, but negatively or very dimly in the two HCC cell lines cultured in the 20 or $1 \% \mathrm{O}_{2}$ conditions. Although comparable expression levels of HIF-1 $\alpha$ were detected between the PDAC and HCC cell lines under $20 \% \mathrm{O}_{2}$, the hypoxic stimulation by $1 \% \mathrm{O}_{2}$ significantly enhanced the expression of HIF- $1 \alpha$ only in the two HCC cell lines, but only slightly upregulated the expression in the PDAC cell lines. b The semiquantitative data are shown
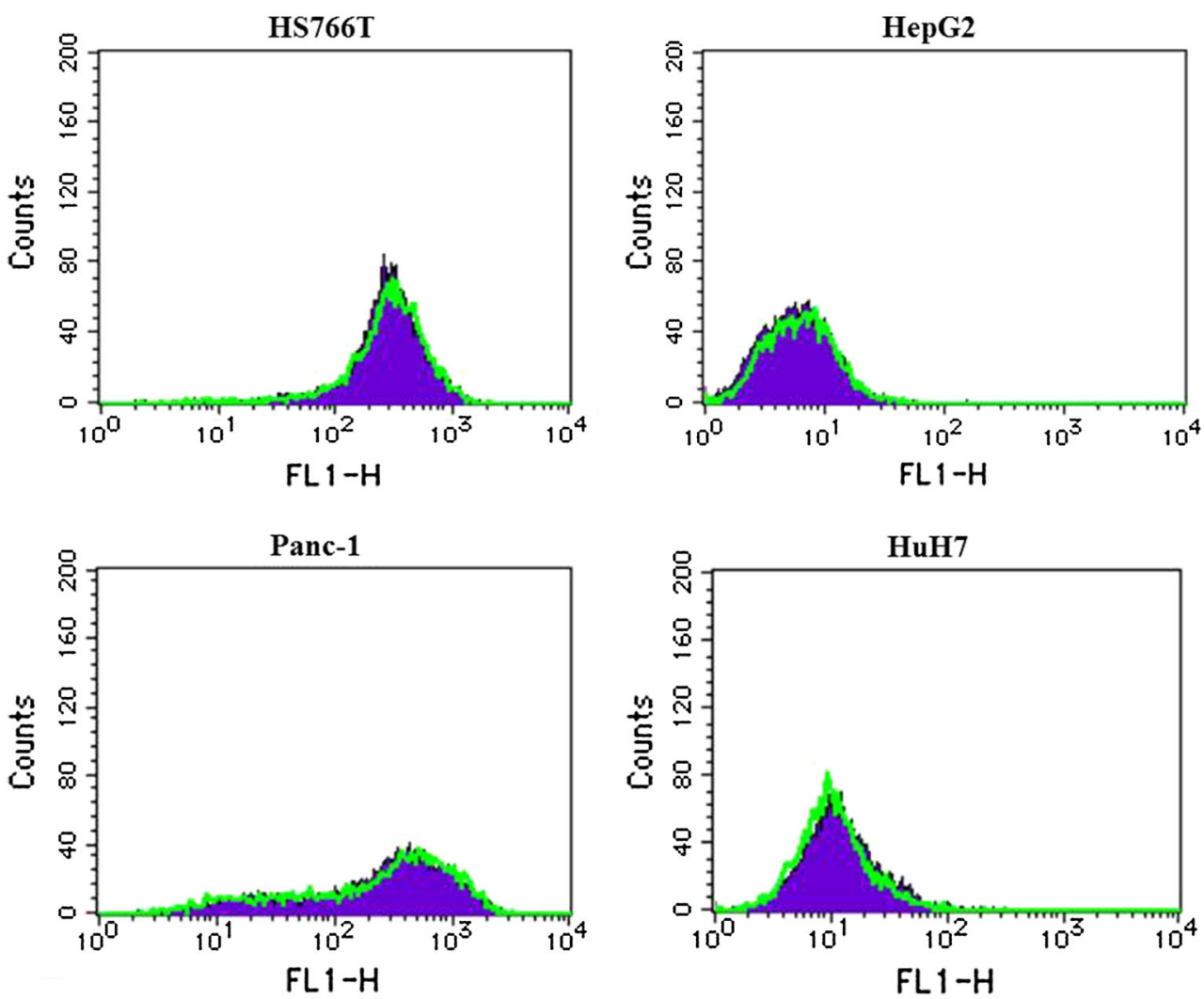

A

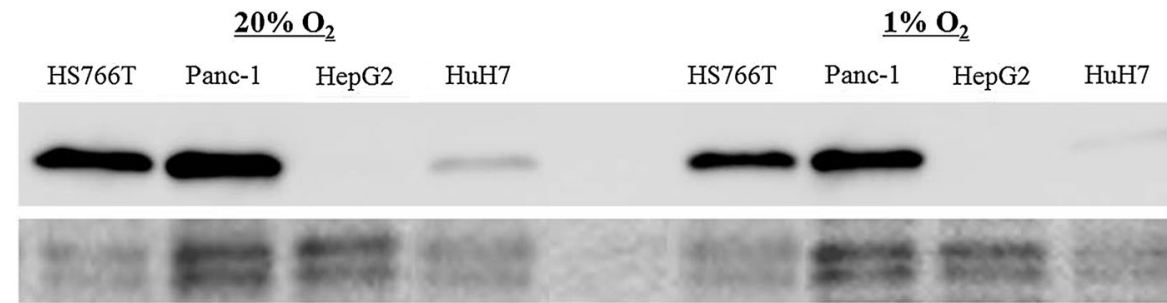

HIF-1 $\alpha$

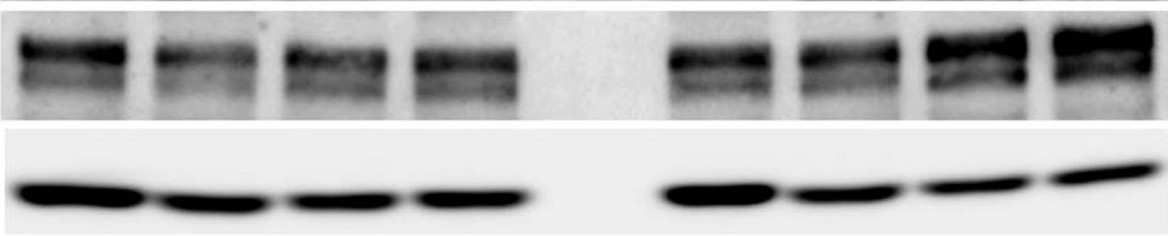

B

PHD3

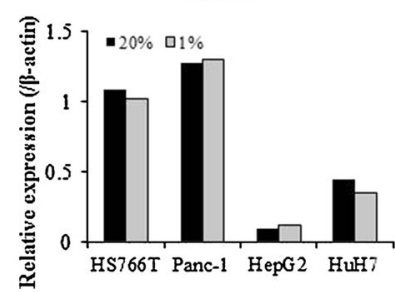

Siah2

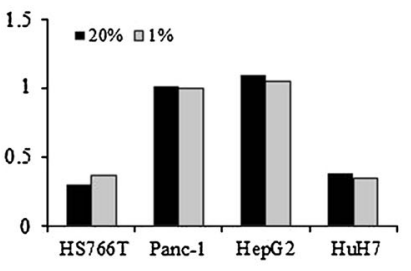

HIF-1 $\alpha$

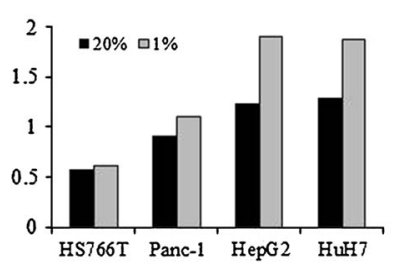




\section{Discussion}

Data from the present study confirmed the poor neovascularization and high expression of the cancer stem cell marker CD44 in PDAC compared to HCC. Our findings also demonstrated for the first time that the increased expression of PHD3 in PDAC tissues likely contributes to the poor neovascularization by repressing the hypoxiainduced HIF-1 $\alpha$ expression and VEGF secretion.

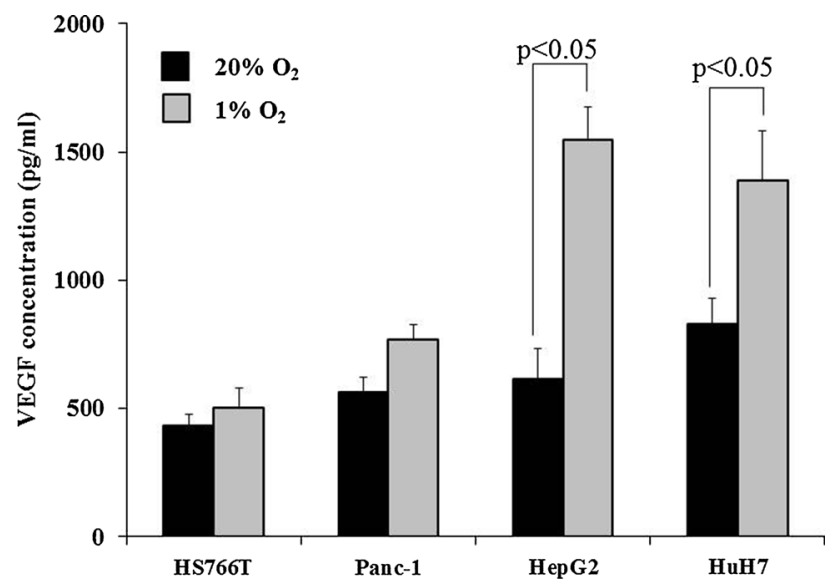

Fig. 5 VEGF secretion from the PDAC (HS766T and Panc-1) and HCC (HepG2 and HuH7) cell lines. The level of VEGF in conditioned medium was measured by ELISA. The secretion of VEGF was comparable between the PDAC and HCC cell lines under $20 \% \mathrm{O}_{2}$. Hypoxic stimulation by $1 \% \mathrm{O}_{2}$ significantly enhanced the VEGF secretion from both of the HCC cell lines $(p<0.05)$, but no significant enhancement was observed in the PDAC cell lines
Intratumoral hypoxia is commonly observed within solid tumor masses [25]. PDAC is well known as one of the most lethal malignant diseases, with the poor responses to various therapies. Previous studies revealed that most cases of PDAC present as a hypovascular tumor mass in medical imaging examinations [1-4], and the oxygen tension was reported to be much lower in the tumor mass of PDAC than in normal pancreas tissue [5]. As tumor hypoxia has been demonstrated to acquire invasive and metastatic properties and to be associated with the rapeutic resistance and increased risk of mortality [6-8], an understanding of the molecular mechanism and tumor biological significance of the poor neovascularization of PDACs may provide new therapeutic strategies for these tumors.

Over the past decade, many studies have identified CSCs, a small subpopulation of cancer cells that preferentially reside in specific hypoxic microenvironments, in various types of solid tumors. These CSCs have also been found to be resistant to radiotherapy and chemotherapy and to be able to form metastatic tumors in other organs $[9,10]$. Taking the characteristics of CSCs into consideration, it seems quite possible that the poor neovascularization in the tumor mass of PDACs may help to enrich CSCs, which are known to contribute to poor prognoses. Our present data showed that the expression of CD44, the most commonly used marker for identifying CSCs was significantly higher in the PDAC samples compared to the HCC samples. Although the sizes of PDAC and HCC tumors are similar, the expressions of CD44 and CD105 varied greatly among
Fig. 6 Knockdown of PHD3 in the PDAC cell line HS766T. a Knockdown of PHD3 was done by siRNA, and the expression levels of PHD3 and HIF- $1 \alpha$ were estimated by Western blot. PHD3 knockdown did not change the expression of HIF- $1 \alpha$ when the cells were cultured in $20 \% \mathrm{O}_{2}$, but the expression of HIF-1 $\alpha$ was clearly enhanced when the cells were cultured in $1 \% \mathrm{O}_{2}$. b The semiquantitative data in HIF- $1 \alpha$ are shown. c Hypoxic stimulation by $1 \% \mathrm{O}_{2}$ significantly enhanced the VEGF secretion from HS766T cells treated by siPHD3 $(p<0.05)$
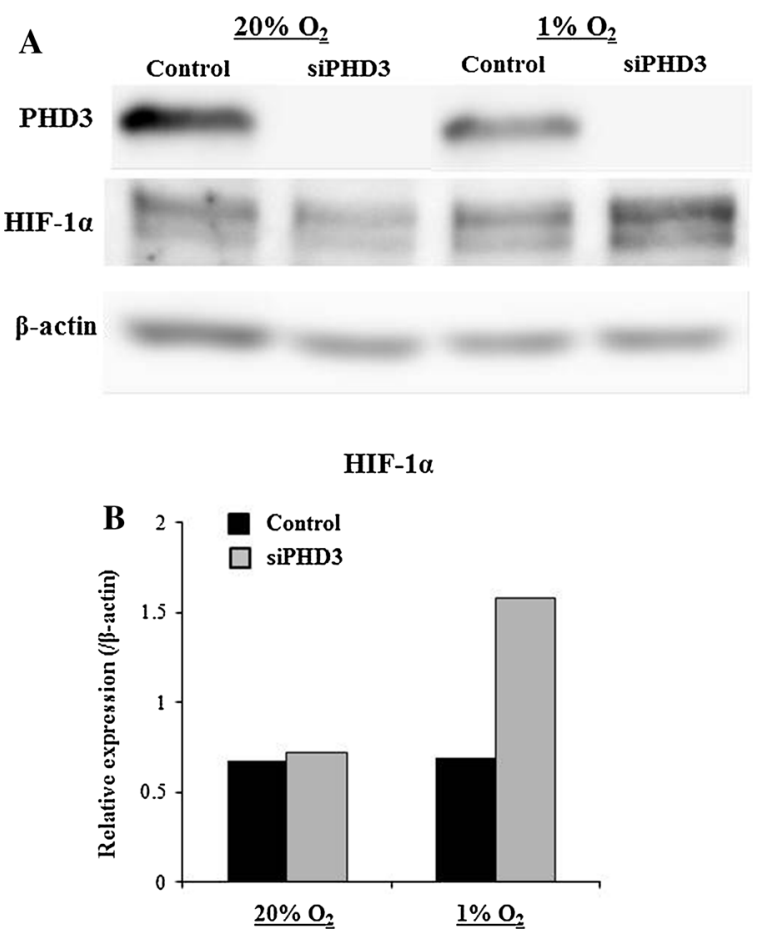

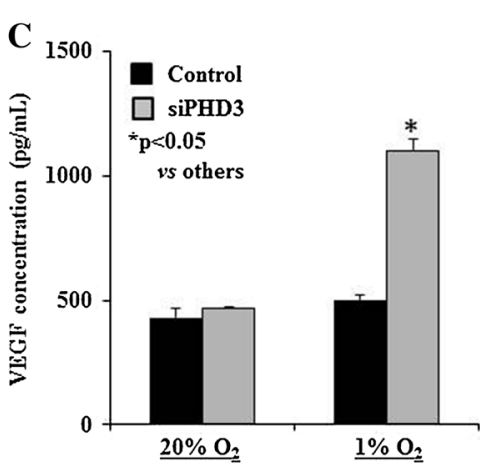


the surgically resected tissue samples of PDAC or HCC in the present study.

Interestingly, the expression of the neovascularization marker CD105 was negatively correlated with CSCs not only in the PDAC tissue samples, but also in the hypervascularized tissue samples of HCC. These immuno-histological findings clearly suggest that the neovascularization of a tumor mass might be able to change the biological property of cancer cells, and that a poorly vascularized tumor mass may induce the enrichment of CSCs. In fact, previous studies found that hypoxic stimulation could enhance the expression of CD44 in various cancer cell lines. Although the expression of CD44 in the present study was much higher in the PDAC cell lines than in the HCC cell lines, hypoxic stimulation did not change the expression of CD44 in any of the cell lines used. Although the reason for this result is unclear, it may be due to the different responses to low oxygen among the cell lines. Further investigation is required to understand the tumor biological significance of poor neovascularization in PDAC.

Hypoxia is known to activate HIF- $1 \alpha$, the central molecule for regulating the oxygen homoeostasis of physiological adaption in response to hypoxia [11-13]. HIF-1 $\alpha$ has also been known to play important roles in neoangiogenesis, epithelial-mesenchymal transition, and stem cell maintenance [6-8]. In agreement with a very recent report [26], the expression of HIF-1 $\alpha$ in the present study's surgically resected PDAC tissues was even lower when compared to that of the well-vascularized HCC tissues. We suspect that the low level of HIF-1 $\alpha$ in the poorly vascularized tumor mass of PDACs might be due to rapid and/or excessive degradation, and we therefore attempted to determine the expression levels of several enzymes that are known to induce HIF-1 $\alpha$ degradation. Compared to the hypervascularized HCCs, the expression of PHD3 (an oxygen-sensitive-type factor for mediating the oxygendependent degradation of HIF-1 $\alpha$ ) was highly and extensively observed in the PDAC tissues. In contrast to PHD3, the weak and negative expression of Siah2 (another enzyme that was recently found to mediate HIF- $1 \alpha$ degradation) was observed in both the PDAC and HCC tissues, although the statistical analysis still showed a significantly lower expression in PDAC than HCC.

By using two randomly selected PDAC cell lines and two HCC cell lines for comparison, we further confirmed the enhanced expression of PHD3 in PDAC by a Westernblot analysis. Siah2 was not clearly detected in any of the cell lines by the Western-blot analysis. Although the expression of HIF- $1 \alpha$ was comparable among all cell lines in the normoxic condition, the hypoxic stimulation induced the significant expression of HIF- $1 \alpha$ and increased the VEGF secretion only in the HCC cell lines, not in the
PDAC cell lines. Hypoxia-induced PHD3 was previously demonstrated to increase HIF- $1 \alpha$ degradation, and it appears to be a direct target of HIF-1 serving as a negative feedback mechanism [18]. Here, although hypoxic stimulation did not induce PHD3 expression in the PDAC cell lines or in the HCC cell lines, the knock-down of PHD3 in one PDAC cell line resulted in the complete recovery of both the hypoxia-induced expression of HIF- $1 \alpha$ and the VEGF secretion.

Together, our findings strongly indicate that an increased expression of PHD3 might contribute to the poor neovascularization of PDACs through the repression of the HIF-1 signaling pathway. Although the tumor biological significance of the poor neovascularization of the tumor mass in PDAC must be further investigated, PHD3 could be a new potential target for the therapeutic resistance of PDACs.

Conflict of interest The authors declare that they have no conflict of interest.

Open Access This article is distributed under the terms of the Creative Commons Attribution Noncommercial License which permits any noncommercial use, distribution, and reproduction in any medium, provided the original author(s) and the source are credited.

\section{References}

1. Hidalgo M. Pancreatic cancer. N Engl J Med. 2010;362:1605-17.

2. Kitano M, Kudo M, Maekawa K, Suetomi Y, Sakamoto H, Fukuta N, et al. Dynamic imaging of pancreatic diseases by contrast enhanced coded phase inversion harmonic ultrasonography. Gut. 2004;53:854-9.

3. Olive KP, Jacobetz MA, Davidson CJ, Gopinathan A, McIntyre D, Honess D, et al. Inhibition of Hedgehog signaling enhances delivery of chemotherapy in a mouse model of pancreatic cancer. Science. 2009;324:1457-61.

4. Pérez-Mancera PA, Guerra C, Barbacid M, Tuveson DA. What we have learned about pancreatic cancer from mouse models. Gastroenterology. 2012;142:1079-92.

5. Koong AC, Mehta VK, Le QT, Fisher GA, Terris DJ, Brown JM, et al. Pancreatic tumors show high levels of hypoxia. Int J Radiat Oncol Biol Phys. 2000;48:919-22.

6. Duffy JP, Eibl G, Reber HA, Hines OJ. Influence of hypoxia and neoangiogenesis on the growth of pancreatic cancer. Mol Cancer. 2003;2:12 (Review).

7. Moncharmont C, Levy A, Gilormini M, Bertrand G, Chargari C, Alphonse G, et al. Targeting a cornerstone of radiation resistance: cancer stem cell. Cancer Lett. 2012;28:139-47.

8. Lu X, Kang Y. Hypoxia and hypoxia-inducible factors: master regulators of metastasis. Clin Cancer Res. 2010;16:5928-35.

9. Koh MY, Lemos R Jr, Liu X, Powis G. The hypoxia-associated factor switches cells from HIF- $1 \alpha$ - to HIF- $2 \alpha$-dependent signaling promoting stem cell characteristics, aggressive tumor growth and invasion. Cancer Res. 2011;71:4015-27.

10. Semenza GL. Hypoxia-inducible factors: mediators of cancer progression and targets for cancer therapy. Trends Pharmacol Sci. 2012;33:207-14. 
11. Wang GL, Jiang BH, Rue EA, Semenza GL. Hypoxia-inducible factor 1 is a basic-helix-loop-helix-PAS heterodimer regulated by cellular $\mathrm{O}_{2}$ tension. Proc Natl Acad Sci USA. 1995;92:5510-4.

12. Semenza GL. Targeting HIF-1 for cancer therapy. Nat Rev Cancer. 2003;3:721-32.

13. Semenza GL. Hypoxia and cancer. Cancer Metastasis Rev. 2007;26:223-4.

14. Luo W, Zhong J, Chang R, Hu H, Pandey A, Semenza GL. Hsp70 and CHIP selectively mediate ubiquitination and degradation of hypoxiainduciblefactor (HIF)-1alpha but not HIF-2alpha. J Biol Chem. 2010;285:3651-63.

15. Ohh M, Park CW, Ivan M, Hoffman MA, Kim TY, Huang LE, et al. Ubiquitination of hypoxia-inducible factor requires direct binding to the beta-domain of the von Hippel-Lindau protein. Nat Cell Biol. 2000;2:423-7.

16. Epstein AC, Gleadle JM, McNeill LA, Hewitson KS, O’Rourke J, Mole DR, et al. C. elegans EGL-9 and mammalian homologs define a family of dioxygenases that regulate HIF by prolylhydroxylation. Cell. 2001;107:43-54.

17. Appelhoff RJ, Tian YM, Raval RR, Turley H, Harris AL, Pugh $\mathrm{CW}$, et al. Differential function of the prolylhydroxylases PHD1, PHD2, and PHD3 in the regulation of hypoxia-inducible factor. J Biol Chem. 2004;279:38458-65.

18. Rantanen K, Pursiheimo J, Högel H, Himanen V, Metzen E, Jaakkola PM. Prolyl hydroxylase PHD3 activates oxygendependent protein aggregation. Mol Biol Cell. 2008;19:2231-40.
19. Marxsen JH, Stengel P, Doege K, Heikkinen P, Jokilehto T, Wagner T, et al. Hypoxia-inducible factor-1 (HIF-1) promotes its degradation by induction of HIF-alpha-prolyl-4-hydroxylases. Biochem J. 2004;381:761-7.

20. Su Y, Loos M, Giese N, Hines OJ, Diebold I, Görlach A, et al. PHD3 regulates differentiation, tumour growth and angiogenesis in pancreatic cancer. Br J Cancer. 2010;103:1571-9.

21. Nakayama K, Frew IJ, Hagensen M, Skals M, Habelhah H, Bhoumik A, et al. Siah2 regulates stability of prolyl-hydroxylases, controls HIF1 $\alpha$ abundance, and modulates physiological responses to hypoxia. Cell. 2004;117:941-52.

22. Al-Hajj M, Wicha, Benito-Hernandez A, Morrison SJ, Clarke MF. Prospective identification of tumorigenic breast cancer cells. Proc Natl Acad Sci USA. 2003;100:3983-8.

23. Collins AT, Berry PA, Hyde C, Stower MJ, Maitland NJ. Prospective identification of tumorigenic prostate cancer stem cells. Cancer Res. 2005;65:10946-51.

24. Dalerba P, Dylla SJ, Park IK, Liu R, Wang X, Cho RW, et al. Phenotypic characterization of human colorectal cancer stem cells. Proc Natl Acad Sci USA. 2007;104:10158-63.

25. Harris AL. Hypoxia-a key regulatory factor in tumour growth. Nat Rev Cancer. 2002;2:38-47.

26. Wang WQ, Liu L, Xu HX, Luo GP, Chen T, Wu CT, et al. Intratumoral $\alpha$-SMA enhances the prognostic potency of CD34 associated with maintenance of microvessel integrity in hepatocellular carcinoma and pancreatic cancer. PLoS One. 2013;8:e71189. 